\section{Microbiological and chemical contamination in different types of food of non-European origin}

\author{
Francesco Casalinuovo, ${ }^{1}$ \\ Vittorio Soprano, ${ }^{2}$ Pasquale Gallo, ${ }^{2}$ \\ Paola Rippa ${ }^{1}$
}

'Istituto Zooprofilattico Sperimentale del Mezzogiorno - Sezione di Catanzaro; 2Dipartimento di Chimica, Istituto Zooprofilattico Sperimentale del Mezzogiorno, Portici (NA), Italy

\section{Abstract}

In the markets of the European Union (EU) the presence of food imported from nonEuropean countries such as Asia, Africa and America is increasingly more widespread. NonEuropean countries, indeed, are much more competitive in terms of prices compared to European countries. For these reasons, EU has issued important laws. The purpose of this study was to assess the effectiveness of these regulations, estimating the levels of microbiological and chemical contamination of food samples of 91 different matrices imported from third countries. The microbiological methods used are those required by the UNI EN ISO, while for the determination of chemical parameters validated methods according to the Standard UNI EN ISO 16140:2003 were used. Our investigation revealed qualitative or quantitative microbial contamination in 23 out of 91 samples analysed (25.2\%). We found high total microbial loads in alimentary conserves, multiple bacterial contamination (Salmonella thiphymurium, Escherichia coli and Vibrio alginolyticus) and viral contamination (Norovirus) in shellfish of the species Cassostrea gigas, and the presence of other pathogens in various products such as hamburgers (Yersinia enterocolitica), frozen fish (Listeria monocytogenes) and honey (Bacillus cereus). With regard to chemical contamination, 24 samples of different food products were analysed. In 9 samples (37.5\%), the levels of the following substances exceeded the permitted limits: histamine (fish conserves) mercury (crab meat), cadmium (crab meat and fish conserves), lead (cheese and honey) and polyphosphates (chicken meat). Despite the limited number of samples analysed, these data prompt reflection on the need to implement a more detailed and rigorous activity of monitoring and control in order to guarantee adequate levels of safety with regard to the consumption of foodstuffs imported into the EU from nonEuropean countries.

\section{Introduzione}

La sicurezza alimentare, definita come la possibilità di garantire in modo costante e generalizzato acqua ed alimenti per soddisfare il fabbisogno energetico di cui l'organismo necessita per la sopravvivenza e la vita, in condizioni igieniche, rappresenta nell'epoca attuale una delle priorità sociali per i paesi industrializzati ed in via di sviluppo (Tantillo et al., 2000). La globalizzazione ed il grande aumento degli scambi commerciali internazionali, ha indotto l'Unione Europea (UE) a creare sistemi di certificazione di origine e di qualità delle produzioni ed in particolare dei prodotti alimentari importati da paesi terzi, in qualche occasione riscontrati qualitativamente inferiori agli analoghi prodotti nazionali (Tantillo et al., 2000). In ambito europeo si è inoltre assistito ad un progressivo aumento dei prezzi e alla contemporanea insufficienza di materie prime alimentari, che hanno determinato un incremento continuo delle importazioni da paesi extraeuropei. In Italia nel 2011 l'incremento è stato del 2,3\% (ISTAT, 2011). Pertanto, in commercio sono sempre più reperibili prodotti alimentari provenienti ormai da ogni parte del mondo e tra questi anche da paesi dove la produttività è caratterizzata da standard igienicosanitari insufficienti, oltre che ad essere basata sullo sfruttamento del lavoro umano. L'UE tutela la salute dei propri cittadini attraverso articolati controlli sulla sicurezza e qualità dei prodotti che arrivano sul mercato europeo e tramite una serie di normative, a cominciare dalla Direttiva 97/78/CE del 18 dicembre 1997 (Commissione Europea, 1997), alla quale hanno fatto seguito altri importanti provvedimenti quali il Regolamento 178/2002/CE (Commis sione Europea, 2002), meglio conosciuto come legislazione alimentare generale e che agli articoli 11-13 della Sezione 3 prevede specifiche disposizioni sullimportazione di alimenti e mangimi nell'UE, il Regolamento 136/2004/CE (Commissione Europea, 2004a), il Regolamento 882/2004/CE (Commissione Europea, 2004b), la Direttiva 104/2006/CE (Commissione Europea, 2006b), il Regolamento 470/2009/CE (Commis sione Europea, 2009) ed il Regolamento 37/2010 (Commissione Europea, 2010), mediante i quali l'Europa ha fissato i principi relativi all'organizzazione dei controlli sanitari e veterinari per i prodotti introdotti da paesi terzi. In Italia, tutte le partite di prodotti provenienti da un paese terzo transitano attraverso i posti d'ispezione frontalieri (PIF) che ne rilascia anche l'autorizzazione all'importazione. I Paesi terzi interessati all'export verso l'UE, coscienti di questa rigorosa politica a difesa dei diritti dei consumatori europei, stanno adeguando le loro filiere produttive ai criteri di sicurezza alimentare e di igiene del processo produttivo previsti dal Regolamento 2073/2005/CE (Commissione Europea, 2005) e successive modifiche (Bagumire et al., 2010). Si
Correspondence: Francesco Casalinuovo, Istituto Zooprofilattico Sperimentale del Mezzogiorno Sezione di Catanzaro, viale Crotone, 88100 Catanzaro, Italy.

Tel. +39.0961.737763 - Fax: +39.0961.738088.

E-mail: francesco.casalinuovo@cert.izsmportici.it

Key words: foodstuffs, imports, microbiological contamination, chemical contamination.

Conflict of interests: the authors declare no potential conflict of interests.

Received for publication: 15 May 2013.

Accepted for publication: 28 May 2013.

This work is licensed under a Creative Commons Attribution 3.0 License (by-nc 3.0).

(C) Copyright F. Casalinuovo et al., 2013 Licensee PAGEPress, Italy

Italian Journal of Food Safety 2013; 2:e36 doi:10.4081/ijfs.2013.e36

è quindi ritenuto interessante condurre una verifica su alcune tipologie di prodotti alimentari di provenienza extraeuropea, regolarmente importati e venduti al dettaglio attraverso la rete commerciale alimentare europea ed italiana. Attraverso controlli analitici di laboratorio è stata valutata la presenza di contaminazione microbiologica e chimica di una serie di alimenti prodotti in paesi terzi, regolarmente importati immessi in commercio attraverso la grande distribuzione dell'UE. Grazie ai costi contenuti e nonostante non siano presenti nei circuiti pubblicitari, a questi prodotti guardano soprattutto i consumatori appartenenti al ceto economico debole, sempre più in difficoltà per via delle congiunture economiche sfavorevoli che da alcuni anni attraversano gran parte del continente europeo. L'indagine ha riguardato 91 diversi campioni di alimenti prodotti in paesi terzi e costituiti da diverse tipologie di matrici quali carni, formaggi, conserve, prodotti della pesca e miele, normalmente acquistati presso supermercati e discount, ma anche in alcuni esercizi commerciali di alimenti cosiddetti etnici sorti per soddisfare la multiforme cultura e tradizione alimentare dell'immigrazione extracomunitaria, tradizioni che stanno diventando delle vere e proprie tendenze anche tra i consumatori occidentali.

\section{Materiali e Metodi}

Sono stati analizzati 91 campioni di diverse tipologie di prodotti alimentari di provenienza extraeuropea, prelevati tutti in confezione originale, congelati interi oppure trasformati o sottoposti a trattamenti (conserve), costituiti da pesce, carne, piatti misti, formaggio, miele, con- 
dimenti e vegetali, riconducibili ad alcune delle categorie alimentari contemplate dal Reg. CE n. 2073/2005 (Commissione Europea, 2005) (Tabella 1). Le zone di provenienza dei prodotti alimentari controllati erano in gran parte riconducibili ai seguenti Paesi terzi: Cina, Marocco, Vietnam, Thailandia, Argentina, Canada, Ecuador, Colombia, Cile, Isole Mauritius, Islanda, Giappone, Filippine, Russia, India e Bangladesh, mentre nel 10\% dei prodotti non era riportata con esattezza la zona di produzione $0 \mathrm{di}$ lavorazione ma solamente generiche aree di provenienza come Oceano Indiano, Oceano Pacifico, Oceano Atlantico nord-orientale. I campioni da sottoporre ai controlli microbiologici e chimici (Tabella 1) sono stati acquistati presso supermercati, discount e rivendite alimentari di prodotti etnici, in confezione originale integra rappresentata da scatole di metallo o involucri di altra natura normalmente utilizzate per il confezionamento degli alimenti. I criteri di scelta dei prodotti erano dettati dalla produzione presso stabilimenti di paesi terzi e dalla presenza in etichetta di informazioni sufficienti per l'identificazione dei costituenti e della data di produzione o scadenza. Le prove microbiologiche hanno riguardato i seguenti parametri e sono state eseguite mediante i rispettivi metodi di prova riportati in parentesi: ricerca di Campylobacter spp. (UNI EN ISO 10272-1:2006) (UNI, 2006), Carica batterica mesofila a $30^{\circ} \mathrm{C}$ (UNI EN ISO4833:2004) (UNI, 2004b), numerazione di Bacillus cereus (UNI EN ISO 7932: 2005) (UNI, 2005a), numerazione di Clostridium perfringens (UNI EN ISO 7937:2005) (UNI, 2005b), numerazione di Coliformi totali (UNI EN ISO 4832:2006) (IS0, 2006), numerazione di Escherichia coli (UNI EN ISO 16649-2:2001) (UNI, 2001), numerazione Enterobacteriacee (UNI EN ISO 215282:2004) (UNI, 2004a), ricerca di Listeria monocytogenes (UNI EN ISO 11290-1:2005) (UNI, 2005d), ricerca di Salmonella spp. (UNI EN ISO 6579:2004) (UNI, 2004d), numerazione
Staphylococcus aureus (UNI EN ISO 68881:2004) (UNI, 2004c), ricerca di Yersinia enterocolitica (UNI EN ISO 10273:2005) (UNI, 2005d), ricerca di Vibrio cholerae e parahaemolyticus (UN EN ISO 21872:2007) (IS0, 2007), ricerca di Norovirus in PCR (MP/CE/005, rev.2 2011). La determinazione della carica mesofila totale sulle conserve in scatola dei prodotti della pesca è stata effettuata in doppio su 2 aliquote diverse del campione precedentemente incubate rispettivamente alla temperatura di $30^{\circ} \mathrm{C}$ per 15 gg e a $55^{\circ} \mathrm{C}$ per $7 \mathrm{gg}$. Inoltre su 24 campioni, rappresentati da 14 prodotti della pesca, 5 prodotti a base di carne, 2 diverse tipologie di formaggi, 2 confezioni di miele e 1 latte caprino UHT, mediante metodi analitici validati secondo la Norma Europea ISO 16140:2003 (ISO, 2003) sono state eseguite anche determinazioni analitiche di tipo chimico per la ricerca di residui di contaminanti e additivi non consentiti 0 non dichiarati, in base alle diverse tipologie di matrici alimentari considerate. È stata quindi effettuata la determinazione qualitativa e/o quantitativa di diversi parametri chimici quali piombo $(\mathrm{Pb})$, cadmio $(\mathrm{Cd})$, ferro $(\mathrm{Fe})$, mercurio $(\mathrm{Hg})$, istamina, ricerca qualitativa solfiti, formaldeide, aflatossina M1, piretroidi, acido ascorbico, fosforo (P), ricerca quantitativa di nitriti e nitrati di sodio, polifosfati, determinazione di proteine di specie, ricerca colorante sudan II-III e IV, ricerca pesticidi fosforati ed organo clorurati. In totale sono state eseguite 1200 prove di laboratorio, utilizzando protocolli analitici sulla base delle diverse tipologie e matrici dei campioni. Sui campioni di conserve in scatola di prodotti della pesca è stata inoltre effettuata la determinazione del $\mathrm{pH}$ in unica misurazione mediante il metodo UNI EN ISO 2917:1999 (ISO, 1999) e l'utilizzo del misuratore Orion Star and Star Plus Meter (Thermo Fisher Scientific, Inc.Waltham, MA, USA) con valore predefinito di 0,01. Per la scelta delle prove chimiche da eseguire è stata considerata la tipologia di matrice da analizzare e la disponibilità di informazioni circa le possibili contaminazioni alimentari di origine ambientale e alla presenza di residui o sostanze vietate nelle derrate alimentari, desumibili dai numerosi Pareri Scientifici del comitato di esperti per conto dell'EFSA e dalla bibliografia scientifica consultata (Draisci et al., 2005; Signorini et al., 2009; Nebbia, 2010)

\section{Risultati}

Nella Tabella 2 sono riportati i campioni risultati non conformi ai criteri microbiologici attuali, mentre nelle Tabelle 3 e 4 sono riportati i campioni risultati non conformi ai criteri chimici rispettivamente per i prodotti della pesca e per altre matrici come preparazioni a base di carne, latte, formaggio e miele.

\section{Prove microbiologiche}

In un campione di Crassostrea gigas, varietà di ostrica proveniente dall'oceano atlantico nordorientale e commercializzata in Italia allo stato fresco, sono stati isolati Salmonella thiphymurium, Vibrio alginolyticus ed i genotipi G1 e G2 di Norovirus. Da sottolineare gli elevati valori di carica batterica mesofila totale riscontrata in un campione di latte bovino pastorizzato, concentrato, intero e zuccherato, la presenza di Yersinia enterocolitica in un hamburger contemporaneamente alla presenza di altri contaminanti quali Staphylococcus epidermidis, Staphylococcus luntus e Rhizobium radiobacter, l'isolamento di Listeria monocytogenes da un campione di pesce congelato, di Bacillus cereus in un campione di miele e di Pasteurella aerogenes in una conserva di sgombro (Scomber scombrus). Tuttavia, il dato più interessante riguarda le condizioni igienicosanitarie generali delle conserve di pesce esaminate, considerati i livelli di carica batterica rile-

Tabella 1. Categorie di prodotti [Reg. (CE) 2073/2005], numero di campioni analizzati e parametri microbiologici e chimici ricercati.

\begin{tabular}{|c|c|c|}
\hline Matrice & Campioni (n) & Parametri ricercati \\
\hline Crostacei e molluschi & 14 & Salmonella spp.; Vibrio spp.; Norovirus; Escherichia coli; Pb; Hg; Cd; Fe; solfiti; istamina \\
\hline Carni e preparazioni a base di carne da consumare cotte & 18 & $\begin{array}{l}\text { Campylobacter spp.; CMT; Bacillus cereus; Escherichia coli; Clostridium perfringens; } \\
\text { Coliformi totali; Enterobacteriacee; Listeria monocytogenes; Salmonella spp.; } \\
\text { Yersinia enterocolitica; Staphilococcus aureus; acido ascorbico; solfiti; proteine; polifosfati; } \\
\text { P; nitriti e nitrati; Sudan }\end{array}$ \\
\hline Prodotti della pesca & 53 & $\begin{array}{l}\text { CMT; Escherichia coli ; Listeria monocytogenes ; Salmonella spp.; Clostridium perfringens; } \\
\text { pH; Vibrio spp.; Pb; Hg; Cd; Fe; solfiti; istamina }\end{array}$ \\
\hline Miele & 2 & CMT; Bacillus cereus; Clostridium perfringens; $\mathrm{Pb}$; Hg; Cd; Fe \\
\hline Latte sottoposto a trattamento termico & 2 & $\begin{array}{l}\text { CMT; Clostridium perfringens; Campylobacter spp.; Staphilococcus Aureus; } \mathrm{Pb} ; \mathrm{Hg} ; \mathrm{Cd} ; \mathrm{Fe} ; \\
\text { formaldeide; aflatossina M1; piretroidi }\end{array}$ \\
\hline Formaggi a base di latte sottoposto a trattamento termico & 2 & $\begin{array}{l}\text { Campylobacter spp.; Bacillus cereus; Escherichia coli; Clostridium perfringens; } \\
\text { Coliformi totali; Enterobacteriacee; Listeria monocytogenes; Salmonella spp.; } \\
\text { Yersinia enterocolitica; Staphilococcus aureus; Pb; Hg; Cd; Fe; formaldeide; aflatossina Ml; } \\
\text { piretroidi }\end{array}$ \\
\hline Totale & 91 & \\
\hline
\end{tabular}

CMT, carica mesofila totale; $\mathrm{Pb}$, piombo; Hg, mercurio; Cd, cadmio; Fe, ferro; P, fosforo. 
vati in 10 prodotti dopo una preincubazione a $30^{\circ} \mathrm{C}$ per $15 \mathrm{gg}$, a volte in associazione con elevati livelli di Coliformi totali (conserva di sgombro in scatola) o caratterizzati dalla presenza di determinate specie batteriche quali Entero coccus columbae (filetti di alici in scatola) e Staphylococcus epidermidis, (sardine in scatola) (Tabella 2).

\section{Prove chimiche}

La Tabella 3 riguarda i campioni di prodotti della pesca risultati non conformi ai criteri chimici per piombo, mercurio, cadmio, ferro, additivi (solfiti) e istamina. Per i metalli pesanti $\mathrm{Pb}$, $\mathrm{Cd} \mathrm{e} \mathrm{Hg}$ i tenori massimi di contaminazione nei prodotti della pesca fanno riferimento al Regolamento CE n. 1881/2006 (Commissione Europea, 2006a), rispettivamente: piombo 0,30$0,50 \mathrm{mgr} / \mathrm{kg}$, mercurio $0,50 \mathrm{mgr} / \mathrm{kg}$ e cadmio $0,05-0,1 \mathrm{mgr} / \mathrm{kg}$. Da segnalare la presenza di valori di istamina di $109 \mathrm{mgr} / \mathrm{Kg}$ in un campione di tonno (thunnus) in scatola, quindi oltre i limiti consentiti di $100 \mathrm{mgr} / \mathrm{Kg}$ (D.Lgs n. 531/1992 e Reg. CE n.2073/2005) (Repubblica Italiana, 1992; Commissione Europea, 2005), mentre in un campione di polpa di granchio ( $c r a b$ meat) sono stati riscontrati valori di $0,874 \mathrm{mgr} / \mathrm{Kg}$ di mercurio, anch'essi oltre il limite consentito di 0,50 mgr/Kg (Reg. CE n. 1881/2006) (Commis sione Europea, 2006a) e valori di 0,2 $\mathrm{mgr} / \mathrm{Kg}$ di cadmio (limite massimo consentito 0,05-0,1 $\mathrm{mgr} / \mathrm{Kg}$, Reg. CE n. 1881/2006) (Commissione Europea, 2006a). Valori di cadmio di 0,14 mgr/Kg e $0,106 \mathrm{mgr} / \mathrm{Kg}$ sono stati inoltre riscontrati rispettivamente in un campione di filetti di sgombro (Scomber scombrus) in scatola e in un campione di tonno (thunnus) in scatola (limite massimo consentito 0,05-0,1 mgr/Kg, Reg. CE n. 1881/2006) (Commissione Europea, 2006a). Infine, significativo appare il valore di 23,5 $\mathrm{mgr} / \mathrm{Kg}$ di ferro riscontrato in un campione di mazzancolle (Penaeus kerathurus), più elevato rispetto al limite ammesso di $18 \mathrm{mgr} / \mathrm{Kg}$ (Istituto Nazionale per la Nutrizione). Nella Tabella 4, relativa a campioni di matrice alimentare diverse dai prodotti della pesca, viene segnalata la presenza di 0,127 mgr/Kg di piombo in un campione di formaggio [limite accettabile 0,02 $\mathrm{mgr} / \mathrm{Kg}$, Reg. CE n. 1881 (Commissione Europea, 2006a) relativamente al latte crudo e latte trattato termicamente], mentre in un campione di miele millefiori erano presenti residui di piombo del valore di $0,259 \mathrm{mgr} / \mathrm{Kg}$. Da segnalare infine il riscontro di polifosfati non dichiarati in carne di pollo lavorata.

\section{Discussione}

Nella valutazione generale dei risultati è necessario tenere in considerazione il numero e la diversità delle matrici analizzate nonché dei parametri microbiologici e chimici verificati. I risultati delle prove di laboratorio effettuate sui 91 campioni alimentari prelevati dal commercio, in generale non rispecchiano un livello igienicosanitario ottimale e, probabilmente, richiamano l'attenzione sulla necessità di maggiori garanzie di qualità e di sicurezza che i paesi terzi, esportatori di derrate alimentare verso il mercato europeo, devono necessariamente essere in grado di garantire ai consumatori italiani ed europei. Si è visto come livelli di contaminazione microbica sono stati rilevati soprattutto nelle conserve alimentari di pesce, nonostante i trattamenti di conservazione a cui sono in genere sottoposti. Per definizione infatti, le conserve dovrebbero essere caratterizzate da sterilità biologica, anche se và sempre più facendosi spazio il concetto di sterilità commerciale, ovvero la considerazione dell'impossibilità che il trattamento termico a cui sono sottoposte le conserve, sia in grado di inattivare il $100 \%$ delle forme microbiche presenti nelle materie prime al

Tabella 2. Campioni risultati non conformi ai criteri microbiologici.

\begin{tabular}{|c|c|c|c|}
\hline Matrice & Campioni (n) & Campioni non conformi (n) & Parametri non conformi \\
\hline Crostacei e molluschi cotti & 14 & Conserva di crostacei (1) & $\mathrm{CMT}=330 \mathrm{ufc} / \mathrm{gr}$ \\
\hline $\begin{array}{l}\text { Carni e preparazioni a base } \\
\text { di carne destinate ad essere } \\
\text { consumate cotte }\end{array}$ & 18 & $\begin{array}{l}\text { Hamburger bovino congelato (1) } \\
\text { Carne bovina congelata (1) } \\
\text { Pasta e carne liofilizzata (2) }\end{array}$ & $\begin{array}{l}\mathrm{CMT}=28800 \text { ufc/gr; presenza di } \\
\text { Yersinia enterocolitica } \\
\mathrm{CMT}=36600 \text { ufc/gr; Coliformi totali }=263000 \mathrm{ufc/gr} \\
\mathrm{CMT}=28600 \text { ufc/gr; CMT }=4770 \text { ufc/gr }\end{array}$ \\
\hline Prodotti della pesca & 54 & $\begin{array}{l}\text { Conserve di pesce scatola } \\
\text { (Thunnus, Engraulis, Sardina, } \\
\text { Scomber, Oncorhynchus) (12) } \\
\text { Molluschi bivalvi (Crassostrea gigas) (1) } \\
\text { Pesce congelato (Pangasius e Oncorhynchus) (2) }\end{array}$ & $\begin{array}{l}\text { CMT=fino a valori di } 4.790 .000 \text { ufc/gr; riscontro di } \\
\text { Pasteurella aerogenes, Enterococcus columbae, } \\
\text { Staphylococcus epidermidis } \\
\text { Presenza di Salmonella thiphymurium (B:4,12:i:1,2), } \\
\text { Norovirus G1 e G2, Vibrio alginolitycus } \\
\text { CMT=fino a } 36000 \text { ufc/gr; Enterobatteri totali= } \\
4900 \text { ufc/gr; Presenza di Listeria monocytogenes, } \\
\text { Staphylococcus spp. }\end{array}$ \\
\hline Miele & 2 & Miele millefiori (1) & CMT $=52900$ ufc/gr; presenza di Bacillus cereus \\
\hline $\begin{array}{l}\text { Latte sottoposto a } \\
\text { trattamento termico }\end{array}$ & 2 & Latte concentrato zuccherato intero (1) & $\begin{array}{l}\mathrm{CMT}=1.000 .000 \mathrm{ufc} / \mathrm{mL} \text { (dopo incubazione a } 30^{\circ} \mathrm{C} \\
\text { per } 15 \mathrm{gg} \text { ) }\end{array}$ \\
\hline Totale & 91 & 22 & \\
\hline
\end{tabular}

CMT, carica mesofila totale; gg, giorni.

Tabella 3. Campioni di prodotti della pesca risultati non conformi ai criteri chimici per piombo, mercurio, cadmio, ferro, additivi (solfiti) e istamina.

\begin{tabular}{|c|c|c|c|c|c|c|}
\hline Matrice & $\begin{array}{c}\mathrm{Pb}^{*} \\
(\mathrm{mg} / \mathrm{kg})\end{array}$ & $\begin{array}{c}\mathrm{Hg}^{*} \\
(\mathrm{mg} / \mathrm{kg})\end{array}$ & $\begin{array}{c}\mathrm{Cd}^{*} \\
(\mathrm{mg} / \mathrm{kg})\end{array}$ & $\begin{array}{c}\text { Solfiti }^{\circ} \\
(\mathrm{mgr} / \mathrm{kg})\end{array}$ & $\begin{array}{c}\text { Fe } \\
\text { (valori normali } \\
18 \text { MGR/kg) }\end{array}$ & $\begin{array}{c}\text { Istamina } \\
\text { (limiti di riferimento } \\
100 \mathrm{mgr} / \mathrm{kg})^{\S}\end{array}$ \\
\hline \multirow{4}{*}{ Prodotti della pesca } & Tonno (Thunnus) in scatola & & 0,106 & & & 109,2 \\
\hline & Sgombro in scatola (Scomber s.) & & 0,146 & & & \\
\hline & Conserva di polpa di granchio & 0,874 & & & & \\
\hline & Mazzancolle congelate (Penaeus) & & & & 23,5 & \\
\hline
\end{tabular}

$\mathrm{Pb}$, piombo; Hg, mercurio; Cd, cadmio; Fe, ferro; P, fosforo. *Limiti di contaminazione da metalli nei prodotti della pesca (in mg/kg) secondo il Reg. CE n.1881/2006 (Commissione Europea, 2006a) (i.e. valori di riferimento): $\mathrm{Pb}=0,30-0,50 \mathrm{mgr} / \mathrm{Kg}, \mathrm{Hg}=0,50 \mathrm{mgr} / \mathrm{Kg}, \mathrm{Cd}=0,05-0,1 \mathrm{mgr} / \mathrm{Kg}$; ${ }^{\circ}$ limiti di contaminazione secondo il DM n. 209 del 27/2/1996 e il DM n.183 del 10/3/2000 (Ministero della Sanità, 1996, 2000); ${ }^{*}$ riferimento: Istituto Nazionale per la Nutrizione; ' D. Lgs n.531/1992 e Reg. CE n. 2073/2005 (Repubblica Italiana, 1992; Commissione Europea, 2005). CMT, carica mesofila totale; gg, giorni. 
Tabella 4. Campioni di latte, formaggio, miele e preparati a base di carne risultati non conformi ad alcuni criteri chimici.

\section{Matrice} Parametro non conforme Valore riscontrato $\mathrm{mgr} / \mathrm{kg}$

Formaggio a base

$\mathrm{Pb}^{*}$

0,127

di latte sottoposto

a trattamento termico (ovino)

\begin{tabular}{lcc} 
Miele & $\mathrm{Pb}^{*}$ & 0,259 \\
\hline $\begin{array}{l}\text { Preparazione a base di carne di pollo } \\
\text { (non dichiarato in etichetta) }\end{array}$ & Polifosfati $^{\circ}$ & $0,89\left(\right.$ come $\left.\mathrm{P}_{2} \mathrm{O}_{5}\right)$
\end{tabular}

$\mathrm{Pb}$, piombo. *Limiti di contaminazione da piombo nel latte crudo e nel latte trattato termicamente $=0,02 \mathrm{mgr} / \mathrm{kg}$ secondo il Reg. CE n.1881/2006 (Commissione Europea, 2006a) (valori di riferimento); limiti di contaminazione secondo il Regolamento (UE) N. 1129/2011 (Commissione Europea, 2011).

momento dell'inscatolamento, soprattutto se presenti in forma sporulata e a livelli iniziali di carica microbica alta. In questo contesto di differente valutazione che implica, da un lato la necessità di garantire la salute dei consumatori attraverso la sicurezza dell'alimento e dall'altro le motivazioni dell'industria conserviera propugnatrice del concetto di sterilità commerciale, sarebbero di valido aiuto poter disporre di limiti microbici di riferimento e di metodi di prova standardizzati per queste tipologie di prodotti alimentari. Il dibattito è più che mai aperto $\mathrm{e}$ l'auspicio è che questo lavoro contribuisca in qualche modo ad evidenziare queste necessità. Occorre inoltre in questi casi pretendere che l'industria conserviera dei paesi terzi sia in grado di garantire e certificare standard produttivi di qualità e di sicurezza alimentare. La presenza di agenti patogeni in grado di determinare episodi di tossinfezioni alimentari quali Listeria monocytogenes, Yersinia enterocolitica, Salmonella thipymurium, Bacillus cereus, dimostrano inoltre come l'esposizione del consumatore al pericolo derivante dal consumo di alimenti provenienti da paesi terzi sia in qualche modo legato ad alcune tipologie di prodotti. In particolare va sottolineato il rischio legato al consumo di molluschi bivalvi crudi o non sufficientemente cotti, come nel caso di Crassostrea gigas, spesso contaminati contemporaneamente da agenti patogeni diversi di natura batterica e virale. A motivo delle ripetute segnalazioni circa le possibili criticità presenti nelle tecniche di coltivazione e di depurazione dei mitili prodotti in particolare aree del sud-est asiatico (Ministero della Salute, 2011c, 2012; Nebbia, 2010) e nonostante le certificazioni sanitarie che accompagno le importazioni sul mercato europeo, si ritiene necessario intensificare i controlli ispettivi ed analitici di questi prodotti sia al momento dell'importazione che durante la fase di commercializzazione. I controlli chimici effettuati sui campioni alimentari considerati nel presente lavoro, hanno rilevato alcune irregolarità relative soprattutto alla presenza di istamina, mercurio e piombo in alcuni dei prodotti controllati, miele compreso, anche se per quest'ultimo non sono disponibili limiti massimi di riferimento ufficiali. Se espresse in termini percentuali, queste irregolarità rappresentano il 37,5\% (9 campioni irregolari su 24 controllati), ovvero un dato che merita una attenta valutazione in quanto potrebbe indicare la necessità di una maggiore incisività dell'attuale attività di sorveglianza $\mathrm{e}$ monitoraggio in atto sul territorio comunitario. $\mathrm{Su}$ alcune confezioni di prodotti, sono state notate non esaustive informazioni relative alla provenienza e composizione dei prodotti controllati; la corretta e completa applicazione delle norme sull'obbligo di rintracciabilità, potrebbe rappresentare un buon deterrente verso tutti gli operatori della filiera produttiva agroalimentare mondiale interessati all'esportazione dei prodotti verso il mercato europeo.

\section{Conclusioni}

Lo studio ha comunque consentito di acquisire importanti informazioni sullo stato igienicosanitario e quindi sul grado di sicurezza di alcune tipologie di prodotti alimentari di provenienza extraeuropea. Per via dei costi inferiori rispetto ad analoghi prodotti europei, a questo tipo di prodotti si rivolgono soprattutto le fasce di consumatori più deboli dal punto di vista economico che, come tutte le altre categorie sociali hanno il diritto ad essere sufficientemente tutelate dal punto di vista della sicurezza alimentare. Per quanto riguarda l'Italia, i risultati dell'indagine contribuiscono quindi a meglio definire le caratteristiche igienico-sanitarie di alcune tipologie di questi prodotti importati da paesi terzi ed allo stesso tempo richiamano la necessità di un controllo più capillare e continuo, magari incrementando i diversi piani già operativi annualmente sul territorio nazionale (Ministero della Salute, 2011a, 2011b), oppure attraverso studi o indagini appositamente commissionati e finanziati, come già avvenuto con successo in altri Stati Membri (Rimstad et al., 2008).

\section{Bibliografia}

Bagumire A, Todd E CD, Nasinyama GW, Muyanja C, 2010. Food safety regulatory requirements with potential effect on exports of aquaculture products from developing countries to the EU and US. African journal of Food Science and Technology 1:31-50.

Commissione Europea, 1997. Direttiva del Consiglio del 18 dicembre 1997 che fissa i principi relativi all'organizzazione dei controlli veterinari per i prodotti che provengono dai paesi terzi e che sono introdotti nella Comunita, 97/78/CE. In: Gazzetta Ufficiale, L24, 30/01/1998.

Commissione Europea, 2002. Regolamento del Parlamento Europeo e del Consiglio del 28 gennaio 2002 che stabilisce i principi e i requisiti generali della legislazione alimentare, istituisce l'Autorita europea per la sicurezza alimentare e fissa procedure nel campo della sicurezza alimentare, 178/2002/ CE. In: Gazzetta Ufficiale, L 31/1, 01/02/2002.

Commissione Europea, 2004a. Regolamento della Commissione del 22 gennaio 2004 che fissa le modalita dei controlli veterinari da effettuare ai posti d'ispezione frontalieri della Comunita sui prodotti importati da paesi terzi, 136/2004/CE. In: Gazzetta Ufficiale, L 21, 28/01/2004.

Commissione Europea, 2004b. Regolamento del Parlamento Europeo e del Consiglio del 29 aprile 2004 relativo ai controlli ufficiali intesi a verificare la conformita alla normativa in materia di mangimi e di alimenti e alle norme sulla salute e sul benessere degli animali, 882/2004/CE. In: Gazzetta Ufficiale, L $165,30 / 04 / 2004$.

Commissione Europea, 2005. Regolamento della Commissione del 15 novembre 2005 sui criteri microbiologici applicabili ai prodotti alimentari, 2073/2005/CE. In: Gazzetta Ufficiale, L 338/1, 22/12/2005.

Commissione Europea, 2006a. Regolamento della Commissione del 19 dicembre 2006 che definisce i tenori massimi di alcuni contaminanti nei prodotti alimentari, 1881/2006/CE. In: Gazzetta Ufficiale, L 364, 20/12/2006.

Commissione Europea, 2006b. Regolamento della Commissione del 20 gennaio 2006 relativo ai titoli d'importazione per i prodotti del settore delle carni bovine originari del Botswana, del Kenia, del Madagascar, dello Swaziland, dello Zimbabwe e della Namibia, 104/2006/CE. In: Gazzetta Ufficiale, L 17, 21/01/2006.

Commissione Europea, 2009. Regolamento del Parlamento Europeo e del Consiglio del 6 maggio 2009 che stabilisce procedure comunitarie per la determinazione di limiti di residui di sostanze farmacologicamente attive negli alimenti di origine animale, abroga il regolamento (CEE) n. 2377/90 del Consiglio e modifica la direttiva 2001/82/CE del Parlamento europeo e del Consiglio e il regolamento (CE) n. 726/2004 del Parlamento europeo e del Consiglio, 
470/2009/CE. In: Gazzetta Ufficiale, L 152/11, $16 / 06 / 2009$.

Commissione Europea, 2010. Regolamento della Commissione del 22 dicembre 2009 concernente le sostanze farmacologicamente attive e la loro classificazione per quanto riguarda i limiti massimi di residui negli alimenti di origine animale, 37/2010/UE. In: Gazzetta Ufficiale, L 15/1, 20/01/2010.

Commissione Europea, 2011. Regolamento della Commissione dell'11 novembre 2011 che modifica l'allegato II del regolamento (CE) n. 1333/2008 del Parlamento europeo e del Consiglio istituendo un elenco dell'Unione di additivi alimentari, 1129/2011/CE. In: Gazzetta Ufficiale, L 295, 12/11/2011.

Draisci R, Falcone E, Patriarca M, Purificato I, Macrì A, 2005. Residui negli alimenti di origine animale: analisi del rischio, prevenzione e sorveglianza. Istituto Superiore di Sanità ed., Roma, Italia. Disponibile al sito: http//www.iss.it/binary/publ/publi/05_C15.1 133788576.pdf

ISO, 1999. Meat and meat products. Measurement of pH. Reference method. Norma ISO 2917:1999. Organizzazione internazionale per la normazione ed., Ginevra, Svizzera.

ISO, 2003. Microbiology of food and animal feeding stuffs. Protocol for the validation of alternative methods. Norma ISO 16140:2003. Organizzazione internazionale per la normazione ed., Ginevra, Svizzera.

ISO, 2006. Microbiology of food and animal feeding stuffs. Horizontal method for the enumeration of coliforms. Colony-count technique. Norma ISO 4832:2006. Organizzazione internazionale per la normazione ed., Ginevra, Svizzera.

ISO, 2007. Microbiology of food and animal feeding stuffs. Horizontal method for the detection of potentially enteropathogenic Vibrio spp. Part 1: Detection of Vibrio parahaemolyticus and Vibrio cholerae. Norma ISO 21872:2007. Organizzazione internazionale per la normazione ed., Ginevra, Svizzera.

ISTAT, 2011. Report on foreign trade 2011. Istituto nazionale di statistica ed., Roma, Italia.

Ministero della Salute, 2011a. Piano nazionale di monitoraggio per la ricerca di microrganismi, loro tossine e metaboliti sugli alimenti di origine animale importati da Paesi Terzi - anno 2011. Ministero della Salute ed., Roma, Italia.

Ministero della Salute, 2011b. Piano nazionale di monitoraggio per la ricerca di residui sugli alimenti di origine animale importati da Paesi terzi - anno 2011. Ministero della Salute ed., Roma, Italia.

Ministero della Salute, 2011c. Relazione sul sistema di allerta comunitario. Ministero della Salute ed., Roma, Italia. Disponibile al sito: http://www.salute.gov.it/imgs/C_17 _pubblicazioni_1890_allegato.pdf

Ministero della Salute, 2012. Relazione sull'attività dei PIF anno 2011. Ministero della Salute ed., Roma, Italia.

Ministero della Sanità, 1996. Decreto Ministeriale $\mathrm{n}^{\circ} 209$ del 27/02/1996. Regolamento concernente la disciplina degli additivi alimentari consentiti nella preparazione e per la conservazione delle sostanze alimentari in attuazione delle direttive $\mathrm{n}$. 94/34/CE, n.94/35/CE, n. 94/36/CE, n. 95/2/CE e n. 95/31/CE. In: Gazzetta Ufficiale, DM 209, 24/04/1996.

Ministero della Sanità, 2000. DECRETO 10 marzo 2000, n.183. Regolamento recante aggiornamento del decreto ministeriale 27 febbraio 1996, n. 209, concernente la disciplina degli additivi alimentari consentiti nella preparazione e per la conservazione delle sostanze alimentari. Recepimento della direttiva 98/72/CE. In: Gazzetta Ufficiale, DM 183, 10/03/2000.

Nebbia C, 2010. Residui di farmaci e contaminanti ambientali. Edises ed., Napoli, Italia.

Repubblica Italiana, 1992. Attuazione della direttiva 91/493/CEE che stabilisce le norme sanitarie applicabili alla produzione e commercializzazione dei prodotti della pesca, tenuto conto delle modifiche apportate dalla direttiva 92/48/CEE che stabilisce le norme igieniche minime applicabili ai prodotti della pesca ottenuti a bordo di talune navi. In: Gazzetta Ufficiale, D.Lgs n. 531/1992, $30 / 12 / 1992$.

Rimstad E, Høiby EA, Kapperud G, Lassen J, Lunestad BT, Nesbakken T, Nygård K, Olsvik $\emptyset$, Robertson L, Tranulis M, Tryland M, 2008. Risk assessment of import and dissemination of intestinal pathogenic bacteria via fresh herbs and leafy vegetables from South-East Asia. Norwegian Scientific Committee for Food Safety ed. Disponibile al sito: http:/www.vkm.no/dav/ 22a5fd4852.pdf

Signorini G, Biagi G, Lucchetti E, Nannipieri S, Marzotto G, Roncaia A, 2009. Residui negli alimenti di origine animale. Ann. Fac. Medic. Vet. Parma 29:77- 90. Disponibile al sito: http://www.unipr.it/arpa/facvet/ annali/2009/biagi.pdf

Tantillo G, Di Pinto A, Vergara A, 2000. Chemical, hygenical and sanitari aspects of italian and foreign honey-proposal of qualitative standars for a "high quality" honey. Ind AlimentItaly 39:981-6.

UNI, 2001. Norma UNI IS0 16649-2:2010. Microbiologia di alimenti e mangimi per animali - Metodo orizzontale per la conta di Escherichia coli beta glucuronidasi-positiva
- Parte 2: Tecnica della conta delle colonie a $44^{\circ} \mathrm{C}$ che utilizza 5-bromo-4-cloro-3- indolil beta-D-glucuronide. Ente Nazionale Italiano di Unificazione ed., Milano, Italia.

UNI, 2004a. Norma UNI ISO 21528-2:2004. Microbiologia di alimenti e mangimi per animali - Metodi orizzontali per la ricerca e la conta di Enterobatteriacee - Parte 2: Metodo della conta delle colonie. Ente Nazionale Italiano di Unificazione ed., Milano, Italia.

UNI, 2004b. Norma UNI EN ISO 4833:2004. Microbiologia di alimenti e mangimi per animali - Metodo orizzontale per la conta di microrganismi - Tecnica della conta delle colonie a $30^{\circ} \mathrm{C}$. Ente Nazionale Italiano di Unificazione ed., Milano, Italia.

UNI, 2004c. Norma UNI EN ISO 6888-1:2004. Microbiologia di alimenti e mangimi per animali - Metodo orizzontale per la conta di stafilococchi coagulasi- positivi (Staphylococcus aureus e altre specie) - Tecnica che utilizza il terreno agar Baird-Parker. Ente Nazionale Italiano di Unificazione ed., Milano, Italia.

UNI, 2004d. Norma UNI EN ISO 6579:2004. Microbiologia di alimenti e mangimi per animali - Metodo orizzontale per la ricerca di Salmonella spp. Ente Nazionale Italiano di Unificazione ed., Milano, Italia.

UNI, 2005a. Norma UNI EN ISO 7932:2005. Microbiologia di alimenti e mangimi per animali - Metodo orizzontale per la conta di Bacillus cereus presunto - Tecnica della conta delle colonie a $30^{\circ} \mathrm{C}$. Ente Nazionale Italiano di Unificazione ed., Milano, Italia.

UNI, 2005b. Norma UNI EN ISO 7937:2005. Microbiologia di alimenti e mangimi per animali - Metodo orizzontale per la conta di Clostridium perfringens - Tecnica della conta delle colonie. Ente Nazionale Italiano di Unificazione ed., Milano, Italia.

UNI, 2005c. Norma UNI EN ISO 10273:2005. Microbiologia di alimenti e mangimi per animali - Metodo orizzontale per la ricerca di Yersinia Enterecolitica patogena presunta. Ente Nazionale Italiano di Unificazione ed., Milano, Italia.

UNI, 2005d. Norma UNI EN ISO 11290-1:2005. Microbiologia di alimenti e mangimi per animali - Metodo orizzontale per la ricerca e la conta di Listeria monocytogenes - Parte 1: Metodo per la ricerca. Ente Nazionale Italiano di Unificazione ed., Milano, Italia.

UNI, 2006. Norma UNI EN ISO 10272-1:2006. Microbiologia di alimenti e mangimi per animali - Metodo orizzontale per la ricerca e la conta di Campylobacter spp. - Parte 1: Metodo per la ricerca. Ente Nazionale Italiano di Unificazione ed., Milano, Italia. 